$\begin{array}{ll}\text { Research Square } & \text { Preprints are preliminary reports that have not undergone peer review. } \\ \text { They should not be considered conclusive, used to inform clinical practice, } \\ \text { or referenced by the media as validated information. }\end{array}$

\title{
In silico analysis to contemplate the chemistry behind gallic acid-mediated inhibition of Polyketide Synthase A from aflatoxin biosynthesis pathway of Aspergillus flavus
}

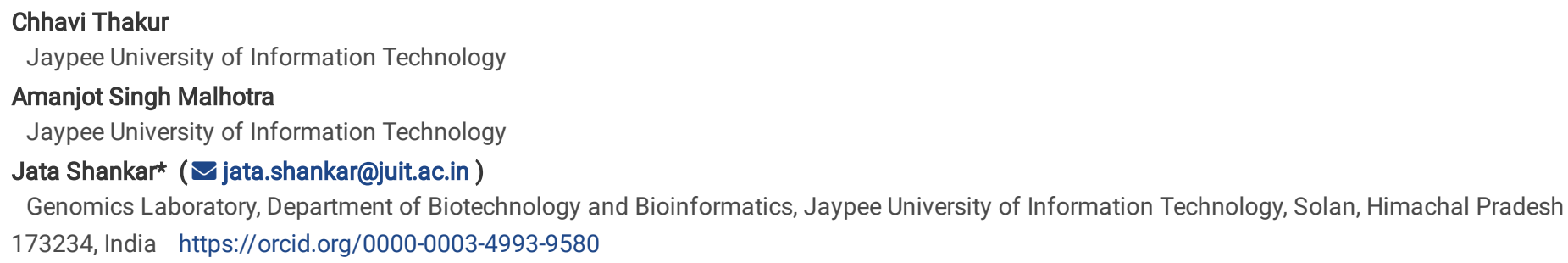

\section{Research Article}

Keywords: Aspergillus flavus, Aflatoxin, Phytochemicals, Gallic acid, Hexanoic acid, Polyketide Synthase A (PksA), Docking, Molecular dynamics simulations, Ligplot, Root Mean Square Deviation

Posted Date: November 9th, 2021

DOI: https://doi.org/10.21203/rs.3.rs-1063934/v1

License: (c) (i) This work is licensed under a Creative Commons Attribution 4.0 International License. Read Full License 


\section{Abstract}

Aspergillus flavus is known for producing the potent carcinogenic agent aflatoxin. Food contamination with aflatoxins is an important safety concern for agricultural yields. To identify and develop anti-aflatoxigenic agents, studies on phytochemicals as anti-aflatoxigenic agents have been documented including gallic acid. Thus, interaction studies using in-silico tools have been explored to understand the molecular mechanism behind inhibition of aflatoxin biosynthesis by studying the chemical interactions of gallic acid with polyketide synthase $\mathrm{A}$ (PksA) of $A$. flavus. The 3D structure of PksA consisting of seven domains was modeled using a Swiss-Model server followed by docking using Autodock tools-1.5.6 with substrate hexanoic acid and with that to gallic acid. The binding energy (electrostatic, inter-molecular or total internal energy) for gallic acid was lower (-6.09 to $-4.79 \mathrm{kcal} / \mathrm{mol}$ ) in comparison to hexanoic acid $(-5.05$ to $-3.36 \mathrm{kcal} / \mathrm{mol})$. During an interaction with the acyl transferase domain of PksA, both ligands showed $\mathrm{H}-\mathrm{bond}$ formation at Glu36, Arg8, Thr11 positions. Ligplot analysis showed the formation of 7-H bonds in gallic acid and 3-H bonds in hexanoic acid. In addition, gallic acid showed stable binding with the active site of PksA indicated by steady root mean square deviation through molecular dynamic simulations. The chemistry between gallic acid and polyketide synthase A(PksA) exhibited that Gallic Acid possesses the highest level of binding potential (more number of hydrogen bonds) with PksA domain in comparison to hexanoic acid, a precursor for aflatoxin biosynthesis. Thus, we suggest enzymes from the aflatoxin biosynthetic pathway in aflatoxin-producing Aspergilli could be an important target for potential inhibitors.

\section{Introduction}

Aspergillus is a genus composed of around 250 recognizable species. ${ }^{1}$ The aspergillum is distinguished by its distinct and unique spore-bearing structure. Aspergillus flavus originally belonged to Flavi's section. This section includes the most critically significant aflatoxin developing fungus-like $A$. flavus and $A$. parasiticus. In this segment, the less prevalent aflatoxin $\mathrm{B} 1$ producing fungus includes $A$. nomius, $A$. parvisclerotigenus, $A$. bombysis as well as $A$. pseudotamarii . ${ }^{2}$ During its colonization of pre-and post-harvest crops leads to aflatoxin production. ${ }^{3}$ Aflatoxins are polyketide-derived secondary metabolites, poisonous and active hepato-carcinogenic agents which are hazardous to animals as well as in humans, it is conventionally an exploitative pathogen for immunocompromised individuals. ${ }^{4}$ These are responsible for approximately $25 \%$ of the entire world food crops is leading to economic losses in developed nations as well as human and animal illness harmed, in developing ones. The development of aflatoxin is the combined effect of fungal life forms, substrates, and the surrounding environment. The factors influencing the development of aflatoxin can be categorized into three parts: nutritional, biological, and physical variables. ${ }^{5}$ Physical parameters like $\mathrm{pH}$, relative humidity as well as moisture, light, aeration, can have an impact on aflatoxin production. ${ }^{6}$ Acetate polyketides are the primary precursors for the secondary metabolite production. The diverse aflatoxins are developed through acetate as well as malonate building blocks formed mostly during idiophase. ${ }^{7}$ The four major key aflatoxins are Cl7 compounds (AFB, AFB2, AFGI, and AFG2) defined as nonaketides. Biosynthesis of aflatoxin includes 23 enzymatic processes and are produced via acetate $\rightarrow$ polyketide $\rightarrow$ anthraquinones $\rightarrow$ xanthones $\rightarrow$ aflatoxins conversion pathway. ${ }^{8}$ At least 15 apparent structurally aflatoxin moieties in the aflatoxin biosynthetic pathway have thus far been established. It's been asserted that 25 genes are involved in aflatoxin biosynthesis and are clustered in a $70-k b$ segment of DNA around the chromosome. ${ }^{9}$ The polyketide biosynthetic pathway causes Aflatoxin biosynthesis in $A$. flavus to be asynchronous process. Polyketide synthase (PKS) is among the essential enzymes in the polyketide biosynthetic pathway. ${ }^{10}$ Fungi corresponding to Type I polyketide synthase comprises extremely significant multifunctional protein, which is $180-250 \mathrm{kDa}$ structured with diverse multiple domains. Ketoacyl synthase, acyl carrier proteins and acyl transferase are the three major domain groups. There have been up to 30 genes and a substantial regulatory gene named aflR gene is involved into aflatoxin biosynthesis involving fatty acid synthases. ${ }^{11}$ Research on Aspergillus nidulans indicated that the generation of secondary metabolite has since been completely rebuilt in mutants whenever mutant of fatty acid synthase itself was processed with hexanoic acid as a precursor. ${ }^{12}$ Polyketides have been identified to be efficacious in decreasing aflatoxin contamination, but there are certain limitations existing along with it. ${ }^{13}$ An alternative approach to fungal contamination is the interface of phytochemicals synthesized from diverse plant sources. ${ }^{14}$ Plants develop these secondary metabolites to provide a defense mechanism against pathogenic fungus. ${ }^{15}$ Due to their antimicrobial properties, they can protect humans and animals against certain diseases induced by microorganisms and even toxins linked with them. ${ }^{16}$ For potential drug research and growth, these metabolites have become the most effective chemo-preventative compounds. ${ }^{17}$ Polyphenols such as gallic acid and quercetin exhibited optimum inhibition against AFB1 production in A. flavus. ${ }^{18}$ Phytochemicals are present in different parts of the plant, such as bark, wood, leaf, fruit, root as well as seed. Since using natural plant products and bio-control additives instead of synthetic chemicals allows scientists to avoid using toxic compounds. ${ }^{19} \mathrm{PksA}$ and its corresponding enzymes from aflatoxin pathway had been identified to be impeded in A. flavus being treated with quercetin. ${ }^{20}$ The results of qRT-PCR indicated that PksA was down regulated, suggesting that quercetin is a strong inhibitor of the polyketide synthase enzyme. Furthermore, In silico study on quercetin or hexanoic acid with every domain of PksA revealed quercetin to be a strong polyketide synthase inhibitor candidate. ${ }^{21}$ Similarly, gallic acid has been demonstrated to have a strong inhibitory effect on aflatoxin production. There is indeed an observation that gallic Acid substantially inhibited the expression of the farB gene, that mostly regulates the activity of peroxisomal fatty acid $\beta$-oxidation and also of the carbon repression regulator encoding gene, creA, that have previously been observed actively engaged in aflatoxin synthesis. ${ }^{22}$ Thus, to understand the gallic acid and PksA interaction, computational methodology could be a valuable tool for researching aflatoxin inhibition mechanisms. ${ }^{23}$ The protein sequence of PksA from A. flavus was extracted from UniProt database to execute homology modeling. Gallic acid has also been docked with the various PksA domains of $A$. flavus, and as compared to the substrate, hexanoic acid, it binds more efficiently. The position of $\mathrm{H}$-bonding and hydrophobic interaction of gallic acid and hexanoic acid with seven distinct domains of PksA was compared using Ligplot analysis. The dynamics of protein-ligand complex formation for every domain was investigated through molecular dynamic simulations. In contrast to hexanoic acid, our results indicated that gallic acid had the highest degree of binding potential with PksA; therefore, gallic acid could inhibit by conservatively binding to the seven domains of PksA, a key enzyme in the aflatoxin biosynthetic pathway. Our study further provided insights into phytochemicals based applications to control aflatoxin contamination in crops.

Page 2/16 
Materials And Methods

\section{Selection of biological data, sequence retrieval, and BLASTp analysis associated with Phylogenetic codification}

From the NCBI Nucleotide Database, the entire amino acid sequence of $A$. flavus polyketide synthase (ATCC 200026/FGSC A1120/NRRL 3357/ JCM 12722/SRRC 167) was retrieved (https://www.ncbi.nlm.nih.gov/)along with NCBI Gene ID of 7914331, gene symbol as well as to Locus tag: AFLA 139410, RNA name: aflC / pksA / pksL1 / polyketide synthase, gene synopsis: aflC/PksA/pksL1/polyketide synthase and UniProt accession number B8NI04 ASPFN. The FASTA amino acid sequence has been used as a query in NCBI BLASTp (http://www.ncbi.nlm.nih.gov/Blast.cgi) to find homologous sequences. The homologous sequences with more than $90 \%$ identification were extracted and matched using MEGA 6.06 tool with muscle analysis (http://www.megasoftware.net/) and then translated into MEGA format. ${ }^{24}$ Phylogenetic tree depicting evolutionary relationships between different biological entities depending upon structural and functional similarities and distinctions was constructed using the maximum likelihood method providing probabilities of the sequences given a model of their evolution. ${ }^{25}$

\section{Ligand preparation}

Ligands were prepared to create their 3-D geometric that include assigning of bond orders, charges and hydrogen atoms to minimize the energy of the structure. Ligand preparation is done before docking for energy minimization as it is used to reduce the overall potential energy of the ligand since biological systems are very dynamic and have low potential energies (-ve delta G) for spontaneous interactions. Less is the energy; more spontaneous will be the interactions. PubChem structure analysis (https://pubch em.ncbi.nlm.nih.gov/) yielded the SMILES string of gallic acid as well as hexanoic acid. The 3D structure of ligands was built using UCSF Chimera for analysis of molecular structures followed by energy minimization

(https://www.cgl.ucsf.edu/chimera/) and Lipinski's law of five (http://www.scfbi o-iitd.res.in/) has been validated. The parameters of the input ligand file were saved in mol2 format. ${ }^{26}$

\section{Independent domain investigation and homology modeling}

The GENE database of NCBI was used to search for seven different PksA domains in the Aspergillus flavus target protein AflC/PksA/pksL1/polyketide synthase (UniProt accession; B8NI04 ASPFN) through extracting desired located sequence from the whole protein sequence for each domain. The conserved nucleotide sequences with their respective locations were incorporated into an online method for converting DNA to proteins(http://insilico.ehu.es/translate/) and translated further into the amino acid template and have been imperiled to homology modelling utilizing SWISS-MODEL server (http://swissmodel.expasy.org/). The SWISS-MODEL server ${ }^{27}$ was used to model all seven domains of PksA and the absolute best model was chosen primarily based on the sequence defined for homology modeling. To identify template structure, the SWISS-MODEL template interface was used. These methods ensure strong alignments at all scales of sequence identity, resulting in the best possible structure prototype. ${ }^{28}$ By sequence identity, the generated models were adapted in our studies. Seven domains of PksA of $A$. flavus, have shown identities between $32.49 \%$ and $99.69 \%$. These protein structures were saved in PDB format for further analysis.

\section{Protein structure preparation and validation}

The stability of Protein structures saved in the PDB format from the swiss-model was endorsed by the PROCHECK web application (http://www.ebi. ac.uk/thornton-srv/). The majority of the protein residues are in the favorable regions, according to the Ramachandran plot analysis for each of the modeled protein structures. ${ }^{29}$ Using UCSF Chimera, protein structure for seven domains were prepared that involves energy minimization, the addition of hydrogen atoms and gasteiger charges.

\section{Molecular docking studies and Ligplot analysis}

The majority of the mechanistic work was conducted in a non-covalent environment, with a molecule (ligand) being incorporated into the desired binding site of a target-specific protein region (receptor) to form a compact complex with potential efficacy and potency. The free binding energy and resilience of complexes were prepared. The docking approach has been used to determine the binding specifications between ligand-receptor complex. ${ }^{30}$ The net expected binding free energy (Gbind) were calculated using parameters such as hydrogen bond (Ghbond), desolvation (Gdesolv), torsional free energy(Gtor), electrostatic (Gelec), dispersion repulsion (Gvdw), and total internal energy (G-total). Autodock tools-1.5.6 (http://autodock.scripps.edu/) was used in the current study to calibrate the docking scores. Gallic acid was docked with seven distinct domains of PksA of $A$. flavus,and evaluated by comparing to the substrate, hexanoic acid. The prepared protein domains were subjected to Autodock software where water molecules were extracted at the beginning and hydrogens were introduced into the proteins accompanied by Kollman charges and Gasteiger charges. AD4 atoms have been allocated and saved in the PDBQT format. Gallic acid and hexanoic acid ligands prepared using UCSF chimera were loaded and saved in PDBQT format. ${ }^{31}$ Grid affinity maps of $126 \times 126 \times 126$ xyz grid points and 1.0spacing were created using the Autogrid program by adjusting the grid covering all of the protein molecules. Rotatable torsions have been unveiled during docking as well as the Lamarckian genetic algorithm was used and 50 runs were performed. To measure van der Waals and electrostatic variables, Autodock parameters were chosen using distance-dependent dielectric functions. Data were collected from 50 different docking trials with each run ends after $25 \mathrm{k}$ energy assessments.Free binding energy, electrostatic energy, constant and final intermolecular energy for inhibition, such as van der Waals, $\mathrm{H}$-bond and desolvation energy were among the research results. ${ }^{32}$ The hydrophobic and $\mathrm{H}$ bond interactions between the ligands and the docked complex receptor were studied using LigPlot software (https://www.ebi.ac.uk/thorntonsrv/software/LIGPLOT/). It was further visualized in 3-D using PyMOL.

Page $3 / 16$ 


\section{Molecular dynamic simulations}

MD (molecular dynamics) is a computer modeling technique for studying the physical movements of molecules and atoms. For a fixed period, the atoms and molecules are allowed to interact, providing a snapshot of the dynamic-evolution of the complex. Atomic and molecular trajectories were calculated using inter atomic potentials or force field molecular dynamics. ${ }^{33}$ Protein-ligand complex was prepared using Pymol tool. Parameterization of proteinligand complex was done using Open-Babel and VMD software (http://www.ks.uiuc.edu/Research/vmd/) to initiate MD simulation by creating topology files for bound ligands. The Solvation Box was created and force field CHARMM was set as a simulation parameter. MD Simulations were run by creating a system and minimizing it using NAMD software (https://www.ks.uiuc.edu/Research/namd/) where the results included Analysis of trajectories, RMSD, RMSF, Hydrogen bonding, and Ramachandran plot which Mimics what atoms do with the potential energy function in real life and analyzes the physical movements of atoms and molecules as provided the positions of the other atoms, the energy equation can be used to measure the force encountered by any atom. ${ }^{34}$

\section{Results}

\section{Homology modeling, sequence alignment, and Phylogenetic analysis}

Using BLASTp, amino acid sequences comparison was carried out. ${ }^{35}$ The BLAST results showed 24 protein sequences (PksA) with more than $90 \%$ identities. Based on query coverage, E-value and similarity ranking, the top 14 sequences were analyzed to construct the phylogenetic tree for PksA of $A$. flavus. The PksA array of $A$. flavus showed related Aspergillus species, primarily A. oryzae, A. parasiticus, A. Pseudo A. nomius, A. novoparasiticus, A. sergii, A. arachidicola, and A. minisclerotigenes (Fig. 1).

In the UCSF chimera, a three-dimensional structure of both ligands including gallic acid and hexanoic acid was developed (Fig. 2).

Figure 2 Three-dimensional structure of gallic Acid (PubChem CID :370) and hexanoic acid (Id: 8892) were obtained from Pubchem and drawn on Chemsketch software

The results of Lipinski's five-rule were evaluated and summarized in Table 1.

Table 1

Lipinski's rule of 5 outlines the molecular properties of ligands

\begin{tabular}{|llllllll|}
\hline Compound & Molecular weight & $\begin{array}{l}\text { H-bond } \\
\text { donor }\end{array}$ & H-bond acceptor & Log P & $\begin{array}{l}\text { Molecular } \\
\text { Refractivity }\end{array}$ & $\begin{array}{l}\text { Pubchem } \\
\text { Cid }\end{array}$ & $\begin{array}{l}\text { Molecular } \\
\text { formula }\end{array}$ \\
\hline Hexanoic acid & $116.16 \mathrm{~g} / \mathrm{mol}$ & 1 & 2 & 1.92 & 125.3074 & 8892 & $\mathrm{C} 6 \mathrm{H} 1202$ \\
\hline Gallic acid & $170.12 \mathrm{~g} / \mathrm{mol}$ & 4 & 5 & 0.7 & 100.4250 & 370 & $\mathrm{C} 7 \mathrm{H} 605$ \\
\hline
\end{tabular}

The NCBI conserved domain sequence database revealed that $A$. Flavus has seven distinct PksA domains. The SWISS-MODEL server has been used to model only those seven PksA domains, and the absolute best model was selected depending upon the sequence specified for homology modelling (Fig. 3).

The SWISS-MODEL Template Library uses the BLAST and HHblits scanning engines to find models and match them with target templates. These methodologies apply to experimental methods by ensuring operational alignments at all levels of sequence recognition, indicating the best template mechanism available for our structure. The sequence of templates was aligned and extracted (Table 2).

Table 2

Seven domains with their respective similarity and sequence identity

\begin{tabular}{|lll|}
\hline Domain name & SMTL Id & Sequence identity \\
\hline ACP Trans acylase domain & 6 fij.1.A & $32.49 \%$ \\
\hline Beta-Ketoacyl Synthase & 6 fij.1.A & $58.78 \%$ \\
\hline Acyl Transferase Domain & 6 fik.1.B & $39.60 \%$ \\
\hline PKS Product Template Domain & 3hrr.1.A & $99.69 \%$ \\
\hline Phosphopantetheine Attachment Site & 2kr5.1.A & $98.53 \%$ \\
\hline Thioesterase Domain & 3ils.1.A & $99.58 \%$ \\
\hline Ketoacyl-synthetase C-terminal & 6 fij.1.A & $55.70 \%$ \\
\hline
\end{tabular}

By analyzing residue-by-residue geometry and overall structure geometry, the stereochemical consistency of a protein structure is tested using Procheck server. The findings showed that 86.7-94.3 percent of all regions were in the most preferred area, 5.3-13.3 percent in the additionally permitted region, 
0.0-1.5 percent in the generously allowed region, and 0-1.4 percent in the disallowed region, according to the Ramachandran plot (Table 3 ).

Table 3

Parameters used for protein structural assessment by PROCHECK analysis (MCBL distribution of main chain bond length, Overall quality factor by ERRAT)

\begin{tabular}{|llllll|}
\hline Domains & Ramachandran Plot (\%) & G factor & MCBL(\%) & Residues & Overall quality factor \\
\hline ACP transacylase domain & $91.9^{\mathrm{a}} ; 6.6^{\mathrm{b}} ; 0.0^{\mathrm{c}} ; 1.4^{\mathrm{d}}$ & $-0.08^{\mathrm{e}} ; 0.15^{\mathrm{f}} ; 0.03^{\mathrm{g}}$ & 91.9 & 238 & 93.33 \\
\hline B-Ketoacyl synthase domain & $88.3^{\mathrm{a}} ; 10.3^{\mathrm{b}} ; 1.1^{\mathrm{c}} ; 0.3^{\mathrm{d}}$ & $-0.25^{\mathrm{e}} ; 0.16^{\mathrm{f}} ;-0.07^{\mathrm{g}}$ & 88.3 & 429 & 89.31 \\
\hline $\begin{array}{l}\text { Ketoacyl synthetase C-terminal } \\
\text { domain }\end{array}$ & $85.3^{\mathrm{a}} ; 13.2^{\mathrm{b}} ; 1.5^{\mathrm{c}} ; 0.0^{\mathrm{d}}$ & $-0.27^{\mathrm{e}} ; 0.13^{\mathrm{f}} ;-0.10^{\mathrm{g}}$ & 85.3 & 79 & 63.04 \\
\hline Acyl transferase domain & $94.3^{\mathrm{a}} ; 5.3^{\mathrm{b}} ; 0.0^{\mathrm{c}} ; 0.4^{\mathrm{d}}$ & $-0.15^{\mathrm{e}} ; 0.14^{\mathrm{f}} ;-0.02^{\mathrm{g}}$ & 94.3 & 299 & 97.53 \\
\hline Product template domain & $89.3^{\mathrm{a}} ; 10.0^{\mathrm{b}} ; 0.7^{\mathrm{c}} ; 0.0^{\mathrm{d}}$ & $-0.31^{\mathrm{e}} ; 0.21^{\mathrm{f}} ;-0.09^{\mathrm{g}}$ & 89.3 & 327 & 88.21 \\
\hline $\begin{array}{l}\text { Phosphopantetheine attachment } \\
\text { site }\end{array}$ & $86.7^{\mathrm{a}} ; 13.3^{\mathrm{b}} ; 0.0^{\mathrm{c}} ; 0.0^{\mathrm{d}}$ & $-0.40^{\mathrm{e}} ;-0.01^{\mathrm{f}} ;-0.2^{\mathrm{g}}$ & 86.7 & 68 & 95.91 \\
\hline Thioesterase domain & & & & 239 \\
\hline
\end{tabular}

Abbreviations used

a- Residue in favored regions

b -Residue in allowed regions

c-Residue in generously allowed regions

d- Residue in disallowed regions

e- $G$ factor score of dihedral bond

$\mathrm{f}-\mathrm{G}$ factor score of covalent bond

g- Overall $G$ factor score

The average goodness factor ( $\mathrm{G}$ factor) was found to be between -0.02 and -0.24 . The ERRAT tool measured the trends of non-bonded interaction of various types of atoms and plotted the magnitude of the error function against the position of the sliding window of 9 residues, which was determined using statistics from highly refined structures. The result indicated the overall quality factors of seven domain structures which ranged from 63.04 97.53. As a result, homology models were used in molecular docking studies based on these validations.

\section{Docking Correlations Validated By Ligplot Analysis}

Gallic acid was showcased to interact with different $A$. flavus domains and was linked to the substrate, hexanoic acid, which is also active in PksA enhancement and aflatoxin biosynthesis control. The prominence-derived dock scores for free binding energy, intermolecular energy, electrostatic energy, and inhibition constant values were compared (Table 4). 
Table 4

Comparative analysis of known protein domains and ligands (gallic acid and hexanoic acid) on target molecule domains

\begin{tabular}{|c|c|c|c|c|c|}
\hline Ligands and Domains & $\begin{array}{l}\text { Binding } \\
\text { energy } \\
\text { (kcal/mol) }\end{array}$ & $\begin{array}{l}\text { Inhibition } \\
\text { Constant } \\
(\mu \mathrm{m})\end{array}$ & $\begin{array}{l}\text { Intermolecular energy } \\
\text { (kcal/mol) }\end{array}$ & $\begin{array}{l}\text { Electrostatic energy } \\
\text { (kcal/mol) }\end{array}$ & $\begin{array}{l}\text { Total internal energy } \\
\text { (kcal/mol) }\end{array}$ \\
\hline \multicolumn{6}{|l|}{ Gallic acid Vs Hexanoic Acid } \\
\hline ACP transacylase domain & $-5.29 /-4.10$ & $131.65 / 994.27$ & $-6.79 /-5.59$ & $-0.28 /-0.27$ & $-1.65 /-0.17$ \\
\hline $\begin{array}{l}\beta \text {-Ketoacyl synthase } \\
\text { domain }\end{array}$ & $-5.33 /-4.87$ & $123.83 / 270.52$ & $-6.82 /-6.36$ & $-0.30 /-0.13$ & $-1.34 /-0.15$ \\
\hline $\begin{array}{l}\text { Ketoacyl synthetase C- } \\
\text { terminal } \\
\text { domain }\end{array}$ & $-4.95 /-3.66$ & $237.22 / 2060$ & $-6.44 /-5.16$ & $-0.39 / 0.44$ & $-1.33 /-0.10$ \\
\hline Acyl transferase domain & $-4.82 /-3.86$ & $295.40 / 1470$ & $-6.31 /-5.36$ & $-0.40 /-0.16$ & $-1.05 /-0.13$ \\
\hline Product template domain & $-5.17 /-4.54$ & $162.91 / 470.42$ & $-6.66 /-6.03$ & $-0.26 /-0.01$ & $-1.68 /-0.09$ \\
\hline $\begin{array}{l}\text { Phosphopantetheine } \\
\text { attachment } \\
\text { site }\end{array}$ & $-4.79 /-3.36$ & $306.33 / 3430$ & $-6.29 /-4.85$ & $-0.81 /-024$ & $-1.03 /-0.10$ \\
\hline Thioesterase domain & $-6.09 /-5.05$ & $34.63 / 197.99$ & $-7.58 /-6.54$ & $-0.41 /-0.17$ & $-1.37 /-0.06$ \\
\hline
\end{tabular}

In all domains, the binding energy of gallic acid was observed to be significantly lower than with hexanoic acid. Gallic acid had binding energy of 6.09 to $4.79 \mathrm{kcal} / \mathrm{mol}$, while hexanoic acid had binding energy of 5.05 to $3.36 \mathrm{kcal} / \mathrm{mol}$ and the electrostatic energy of gallic acid $(0.81$ to $0.26 \mathrm{kcal} / \mathrm{mol})$ and hexanoic acid ( 0.44 to $0.01 \mathrm{kcal} / \mathrm{mol}$ ) are highly comparable. Comparative Analysis on the basis of binding energy is shown in Fig. 4 .

Intermolecular energy is directly proportionate to binding energy, which was between 7.58 and $6.29 \mathrm{kcal} / \mathrm{mol}$ for hexanoic acid and between 6.54 and $4.85 \mathrm{kcal} / \mathrm{mol}$ for hexanoic acid. A reduction in intermolecular energy was observed along with final internal energy which is in the range of -1.68 and -1.03 in gallic acid and -0.17 and -0.06 in hexanoic acid, implying that Gallic acid is a more effective binder with these domains. This correlative appraisal for gallic acid and hexanoic acid interplay with seven varying domains of PksA was systematically summarized (Fig. 5).

The Hydrogen bonding and hydrophobic active sites of Gallic acid, as well as hexanoic acid with seven main domains of PksA, were identified and compared using Ligplot tools. Out of seven domains, Both gallic acid and hexanoic acid showed the maximum inhibition with Thioesterase Domain and lowest inhibition potential with Phosphopantetheine attachment domain. Gallic acid formed 7-H bonds, while hexanoic acid formed 3-H bonds, according to Ligplot research. When the acyltransferase domain interacts substrate such as gallic acid and hexanoic acid showed H-bond formation at Glu36, Arg8, Thr11 positions. Also, in Phosphopantetheine attachment domain, gallic acid form 6-H bonds in contrast to the 1-H bond in hexanoic acid. In ACP, TH, KS, PT Domains, No. of H-bonds were observed more as compared to gallic acid and hexanoic acid. In AT, PP, CT domains, gallic acid was observed to have more $\mathrm{H}$-bonds than hexanoic acid. Since in correlation to hexanoic acid, gallic acid showed the maximum binding long-term potential (maximum proportion of Hydrogen bonding) well with PksA domain; therefore, gallic acid can inhibit by collegiately binding well to polyketide synthase domains. This comparative analysis was evaluated (Table 5). 
Table 5

Comparative analysis on the basis of hydrophobic and hydrogen bonding of gallic acid and hexanoic acid with different PksA domains of Aspergillus

\begin{tabular}{|c|c|c|c|c|c|}
\hline $\begin{array}{l}\text { Protein } \\
\text { Domains }\end{array}$ & Ligands & $\begin{array}{l}\text { No. of } \\
\text { Hydrogen } \\
\text { bonds }\end{array}$ & Hydrogen bonding & Bond length 0 & Hydrophobic bonding \\
\hline \multirow[t]{2}{*}{ ACP } & GA & 4 & Leu70,GIn6 & $2.95,2.90,2.81,2.75$ & Arg75,Tyr65, Pro232 \\
\hline & $\mathrm{HA}$ & 3 & Tyr65, Arg75, Gln6 & $2.90,2.87,2.80$ & Gly68,Leu70,Pro232 \\
\hline \multirow[t]{4}{*}{ CT } & GA & 5 & Glu36,Arg8, & $3.21,3.07,2.98,2.96,2.73$ & Glu32,Ala34,Pro12,Asp33, \\
\hline & & & Thr11 & & His10,Pro35 \\
\hline & $\mathrm{HA}$ & 3 & Glu36,Arg8, & $3.09,3.03,2.63$ & Pro12,Pro35,Ala34,Asp33, \\
\hline & & & Thr11 & & CHis10 \\
\hline \multirow[t]{4}{*}{ TH } & GA & 4 & Asp174,His222, & $3.11,2.68,2.79,2.72$ & Lys194,Thr175,Asn221,Phe190,Met191,Asp98 \\
\hline & & & Arg195 & & ,Gln193 \\
\hline & $\mathrm{HA}$ & 1 & Phe190 & 3.32 & Thr175,GIn193,Val76,Asp98,Met191,Ala173,His222, \\
\hline & & & & & Ala172,Asp174,Arg195,Lys194 \\
\hline \multirow[t]{3}{*}{ PP } & GA & 6 & Glu66,Arg63, & $2.74,3.00,2.55,2.89$ & Met4,Val62,Thr19 \\
\hline & & & Leu18,Asp20 & $3.26,2.70$ & \\
\hline & HA & 1 & Arg63 & 2.87 & Val62,Asp20,Glu66 \\
\hline \multirow[t]{4}{*}{ KS } & GA & 3 & Ser12,Trp60, & $3.15,2.79,2.64$ & Arg8, Leu61, Glu11, Phe63, Pro13, Phe9 \\
\hline & & & Asp62 & & \\
\hline & HA & 2 & Ile258 & $2.89,2.76$ & Lys242, Leu183, Ile241, Val257, Trp184, Arg243 \\
\hline & & & & & Cys180, Ala1 \\
\hline \multirow[t]{3}{*}{ PT } & GA & 3 & Phe227,His230, & $3.10,3 \cdot 05,2.70$ & His233, Ala228, Thr226 \\
\hline & & & Leu272 & & \\
\hline & HA & 0 & - & - & $\begin{array}{l}\text { Met187, Gly183, Leu200, Arg180, Leu203, Tyr178, } \\
\text { Phe243, Tyr184, Tyr202 }\end{array}$ \\
\hline \multirow[t]{4}{*}{ AT } & GA & 7 & $\begin{array}{l}\text { His246,His196,Ser93,Leu94,GI } \\
\text { 65,Arq118 }\end{array}$ & 2.92,2.92,3.03,3.16, & Leu122,Phe195,Cys247 \\
\hline & & & & $2.92,2.57,3.12$ & \\
\hline & HA & 3 & Gln8,Leu94, & $2.59,3.05,3.15$ & Phe195,His246,His196,Arg118,Gly7 \\
\hline & & & Ser93 & & \\
\hline
\end{tabular}

Abbreviation used:

GA - Gallic acid

HA - Hexanoic acid

ACP - ACP Transacylase domain

CT - Ketoacyl Synthetase C-terminal domain

TH- Thioesterase Domain

PP - Phosphopantetheine attachment site

KS - Beta Ketoacyl Synthase domain

PT - Product template domain

AT- Acyl Transferase domain

\section{Simulation}


To gain a better understanding of protein binding affinity and to obtain dynamic insights into complex systems, molecular dynamic simulations has been conducted. MD simulations were conducted on the processed hits from molecular docking experiments of gallic acid and hexanoic acid as reference inhibitors by using VMD as a molecular visualization software that uses 3-D graphics and integrated scripting to view, animate, and analyze large biomolecular structures of protein and ligand and NAMD software has been a parallel molecular dynamics code that uses the CHARMM27 force field as a simulation parameter for elevated simulation of complex bio-molecular systems. Simulations of molecular dynamics are used to model the diverse behaviour of molecular systems over time. This system makes it possible for researchers to better comprehend the versatility and complexities of proteindrug binding. For every chosen molecule, a specific simulation system was developed. Open-babel software was used to create simulation parameters for all ligands. Simulations were run in a solvation box with its periodic boundary conditions with systems neutralized by incorporating counter ions to it. To prevent steric clashes, an energy minimization component to every system was performed employing 5000 steps of the steepest descent algorithm and a total force of Thousand kJ/mol. The PME grid size was set $100 \times 100 \times 100$ grid points and the temperature was set as 310 and molecular dynamic simulations were run for 5000 steps. The root mean square value of Backbone atoms of proteins and energy profiles that can be measured using simulations of both gallic acid and hexanoic acid showed that the simulated systems are fairly stable(Table 6) .

Table 6

Simulation of Gallic Acid

\begin{tabular}{|llllll|}
\hline DOMAINS & AVG & STSD & MIN & MAX & NUM \\
\hline ACP & 1.88 & 0.68 & 0.09 & 3.99 & 102 \\
\hline AT & 2.15 & 0.58 & 0.09 & 0.89 & 102 \\
\hline CT & 1.35 & 0.24 & 0.04 & 1.58 & 102 \\
KS & 0.80 & 0.13 & 0.05 & 0.94 & 102 \\
\hline PP & 0.97 & 0.19 & 0.04 & 1.17 & 102 \\
\hline PT & 1.73 & 0.45 & 0.05 & 3.21 & 102 \\
\hline TH & 0.83 & 0.15 & 0.03 & 0.98 & 102 \\
\hline
\end{tabular}

Avg, max, min, stdev and num \&gt; When using the -all frames argument, this specifies the rule to use to merge frames. These correspond to retaining the average, maximum, and minimum values from the measured frames\&\#39; set. The standard deviation for each point over the range of frames will be returned by stdev and num specifies the no. of atoms selected.

The RMSD values in the case of gallic acid were between $0.80 \mathrm{~nm}$ and $2.15 \mathrm{~nm}$ for PksA-gallic acid complexes and $0.62 \mathrm{~nm}$ and $1.47 \mathrm{~nm}$ for PksAhexanoic acid complexes. Shifting RMSD values within the first few nanoseconds of simulations demonstrate the initial alteration of ligands at the active site of PksA. RMSD measurements were performed for the chosen molecule via the selected frames with a reference point of both specified molecule and its respective frame. The calculation's results appeared in the results list, where these are plotted in graphs (Fig. 6).

The results list contains details about the RMSD calculations that have been completed. A dotted line between the two atoms if there is indeed a potential hydrogen bond among both of them (Fig. 7).

The results of this analysis revealed that each simulation model obtained relatively stable potential energy profiles that remained constant during the simulation cycle. These results suggest that all protein-ligand complex structures are stable and consistent, paving the way for further research into binding modes, important molecular interactions, and free energy calculations. As a result, the RMSD, hydrogen bonding and heat map representation depicted gallic acid to form more Stable RMSD of protein backbone atoms as well as significant upside energy profiles in comparison to hexanoic acid.

\section{Discussion}

Aspergillus flavus usually colonize on wide spectrum of crops and mostly in prevalent soil and air environment. In agriculture, the primary concern with such a fungus is that it induces pharmacologically active toxins called aflatoxin which is carcinogenic in nature and a health threat for animals. In human beings, it is extensively a deceitful pathogen for immunocompromised individuals. ${ }^{3}$ Fungal species like $A$. flavus, $A$. nomius as well as A.parasiticus afflict plant species throughout development, growing and preservation are responsible for producing these hazardous toxins. These toxins can endure food production and are recognized being an unpreventable food contaminant by the Food and Drug Administration. ${ }^{36}$ Synthetically, aflatoxins are derivatives of difuranocoumarin formed via a polyketide pathway. The key four toxins among sixteen structurally analogous toxins are the G1-G2 and B1-B2 aflatoxins. ${ }^{37}$ These characters correspond towards both colors underneath ultraviolet light (green or blue) of its fluorescence and to the figures showing their distance measure of mobility on the chromatographic slender plate. The main naturally produced formidable potent is Aflatoxin B1 as a carcinogenic agent. ${ }^{38}$ The multi-domain polyketide Synthase A (PksA) enzyme encompasses a hexanoyl starter unit including seven malonyl-CoA adhesive units, which initiate aflatoxin biosynthesis by synthesizing the protonated norsolorinic acid anthrone in the dynamic aflatoxin B1 pathway. Aflatoxins' biosynthesis genes, as well as those of many secondary metabolites, have been grouped. ${ }^{39}$ The whole cluster has also undergone sequencing and transcription. ${ }^{40}$ The cluster of genes is $82-\mathrm{kb}$ long as well as incorporates twenty-five diversified genes. Comprehending aflatoxin biosynthesis is anticipated to boost the creation of control methods that provide an insight into why and how the aflatoxin has been formulated. The genome of $A$. flavus was lately sequenced. ${ }^{41}$ The data thereby establish significant tools for comprehension of both fungus and aflatoxin production. Prevention 
seems to be the ideal solution to limiting the toxicity of aflatoxins Susceptibility to agricultural crop production. Phytochemicals are under consideration in comparison to conventional compounds to establish approaches to combat fungal diseases globally from the past few years. The term "phytochemicals" is assigned to naturally produced, non-nutritive bioactive organic compounds of plant sources that have resistant and disease preventive characteristics. ${ }^{42}$ Many phytochemicals have become detrimental to fungi but can be utilized to protect crops, livestock, humans, food, and feed from toxic fungi. And hence, it's indeed vital to examine a feasible, cost efficiency, and non-toxic mechanism for the preventative measures for fungal degradation thereby creating an opportunity to dissuade artificial preservatives implemented by the utilization of natural plant materials and inhibitor derived from these plant sources. Experimental data demonstrates gallic acid exemplifies anti-aflatoxigenic and anti-Aspergillus functions. ${ }^{22} \mathrm{~A}$ contingent of Tulare walnut pellicle, gallic acid (GA), clearly indicates a significant inhibition effect against aflatoxin biosynthesis. ${ }^{43}$ In-vitro analysis on gallic acid showed significant inhibition against $A$. flavus PksA. As a result, a computationally integrated study with PksA protein of $A$. flavus was carried out to understand the mechanisms involved underlying the Phytochemicals suppression of aflatoxin biosynthesis. ${ }^{21}$ In our analysis, gallic acid, as well as hexanoic acid as a precursor, exhibited similar binding properties by forming a single H-bond at Phe190 Position and 4-H bonds at Asp174, His222, Arg195 positions. It also indicates a greater binding affinity for gallic acid comparable to hexanoic acid as the gallic acid's binding energy was found to be $-6.09 \mathrm{kcal} / \mathrm{mol}$ in correlation with hexanoic acid $-5.05 \mathrm{kcal} / \mathrm{mol}$ binding energy in the thioesterase domain of PksA. Hydrophobic interactions indicated that seven amino acids were involved in gallic acid and eleven amino acids were involved in hexanoic acid. Photochemical showed stable binding with the active site of PksA demonstrated by persistent RMSD and reliable energy profiles of protein backbone atoms. In the interaction with gallic acid, the amino acids Arg75 and Tyr65 were found in the hydrophobic region although precluded in the interplay of hexanoic acid with the ACP trans acylase domain as Gly68, Leu70 are involved with one common residue Pro232 in Hydrophobic bonding. This implies that the ACP transacylase domain of PksA has distinct binding sites for gallic acid and hexanoic acid. Gallic acid's binding specificity with the ACP transacylase domain, on the other hand, was found to be greater than hexanoic acid's in terms of binding energy $(-5.29 \mathrm{kcal} / \mathrm{mol} \mathrm{vs}$. $-4.10 \mathrm{kcal} / \mathrm{mol})$ and formation of $7-\mathrm{H}$ bonds in gallic acid and 3-H bonds formation in hexanoic acid. In contrast to hexanoic acid, in silico techniques revealed active binding of gallic acid with product template domain. Thus, gallic acid is more stable due to the formation of 3-H bonds at Phe227, His230, and Leu272 positions in product template domain and no bond formation in hexanoic acid. In addition, among the 7 domains of PksA, both ligands had the lowest electrostatic energy

$(-0.26 /-0.01)$ when they interacted with the product template domain. Gallic acid showed the highest Intermolecular energy as compared to hexanoic Acid (-1.68 /-0.09) with product template domain. In ketoacyl synthetase C-terminal domain-PksA complex, both gallic acid and hexanoic acid show hydrogen bonding at Glu36, arg8, thr11 positions but CT-PksA with gallic acid is more stable due to the formation of 5-H bonds in gallic Acid as compared to 3-H bonds in hexanoic acid. Among the 7 domains, the acyltransferase domain and phosphopantetheine attachment site showed the highest number of hydrogen bonding with gallic acid i.e., $7 / 6$ and 3/1 in hexanoic acid, respectively. According to our findings, gallic acid has a stronger binding affinity in these two domains than hexanoic acid, since GA needs less binding energy for AT $(-4.82 \mathrm{kcal} / \mathrm{mol})$ as well as PP $(-4.79 \mathrm{kcal} / \mathrm{mol})$ whereas hexanoic acid requires $-3.86 \mathrm{kcal} / \mathrm{mol}$ and $-3.36 \mathrm{kcal} / \mathrm{mol}$, respectively. Natural components of polyketide undergo various frameworks and biochemical processes and combine with fatty acid synthesis, a subsection of biosynthetic measures. Thioesterase most frequently performs the penultimate metamorphosis catalyzed by polyketide synthases as well as fatty acid synthases. In silico studies of gallic acid's interaction with the thioesterase domain of $A$. flavus, PksA, revealed that gallic acid had greater binding energy than hexanoic Acid $(-6.09 \mathrm{kcal} / \mathrm{mol} \mathrm{vs}-5.05 \mathrm{kcal} / \mathrm{mol}) \mathrm{validated} \mathrm{by}$ stronger hydrophobic interactions and 4-H bonds formation at Asp174, His222, Arg195 positions in GA as compared to 1-H bond in HA at Phe190 position suggesting among 7 domains. As a result, thioesterase domain plays a key role in inhibiting aflatoxin biosynthesis in A. flavus. Also $\beta$-Ketoacyl synthase, The PksA domain demonstrated more extensive binding for gallic Acid than hexanoic acid in respect of binding ability, including $\mathrm{H}$-bonding and hydrophobic interactions. The molecular docking and molecular dynamic simulation of both gallic acid and hexanoic acid with every domain of PksA revealed gallic acid as a promising target for polyketide synthase inhibition based on in silico analysis utilizing the 3-D structure of seven domain categories of $A$. flavus and evaluating their properties which include hydrogen bonding and hydrophobic interactions along with its binding affinity and electrostatic energy.

\section{Conclusion}

Docking scores such as binding free energy, intermolecular energy, electrostatic energy and inhibition constant value results revealed that the binding energy of gallic acid was less than that of hexanoic acid in all the respective domains. The binding energy of gallic acid ranged between - 6.09 and $4.79 \mathrm{kcal} / \mathrm{mol}$ and that ofhexanoic acid between -5.05 and $-3.36 \mathrm{kcal} / \mathrm{mol}$. Out of these seven domains, Both gallic acid and hexanoic acid showed the maximum inhibition with Thioesterase Domain. LigPlot analysis showed the formation of 7-H bonds in gallic acid and 3-H bonds in hexanoic acid. Phytochemicals showed stable binding with active site of PksA indicated by steady RMSD of protein backbone atoms and potential energy profiles. Our results indicated that gallic acid exhibited the highest level of binding potential with PksA domain named Thioesterase domain in comparison to hexanoic acid; thus, gallic acid feasibly inhibits by competitively binding to the seven domains of PksA, a critical enzyme of aflatoxin biosynthetic pathway. Our study may find its application in phytochemicals based inhibitors for aflatoxin pathway.

\section{Declarations}

Author contribution: CT, JS conceived and designed the research articles. CT and ASM carried the work. JS contributed materials or analytical tools and supervised the work. CT and JS wrote the manuscript. All authors read and approved the manuscript. 


\section{Acknowledgment: We are thankful to the Department of Biotechnology \& Bioinformatics, Jaypee University of Information Technology, Solan, HP for providing facilities.}

Compliance with ethical standards

\section{Conflict of interest: The authors declare that they have no conflict of interest.}

\section{Ethical approval: This article does not contain any studies with human participants or animals performed by any of the authors.}

\section{References}

1. V. Meyer, B. Wu and A. F. J. Ram, Biotechnology Letters, 2011, 33, 469-476.

2. J. C. Frisvad, P. Skouboe and R. A. Samson, Syst Appl Microbiol, 2005, 28, 442-453.

3. M. A. Klich, Mol Plant Pathol, 2007, 8, 713-722.

4. M. T. Hedayati, A. C. Pasqualotto, P. A. Warn, P. Bowyer and D. W. Denning, Microbiology (Reading), $2007,153,1677-1692$.

5. U. P. Sarma, P. J. Bhetaria, P. Devi and A. Varma, Indian J Clin Biochem, 2017, 32, 124-133.

6. T. K. Patel, R. Anand, A. P. Singh, J. Shankar and B. N. Tiwary, Biotechnology and Bioprocess Engineering, 2014, 19, 1105-1113.

7. P. Kumar, D. K. Mahato, M. Kamle, T. K. Mohanta and S. G. Kang, Front Microbiol, 2016, 7, 2170.

8. J. Huffman, R. Gerber and L. Du, Biopolymers, 2010, 93, 764-776.

9. J. W. Cary, N. Barnaby, K. C. Ehrlich and D. Bhatnagar, App/ Microbiol Biotechnol, 1999, 51, 808-812.

10. J. M. Crawford and C. A. Townsend, Nat Rev Microbiol, 2010, 8, 879-889.

11. J. Yu, Toxins (Basel), 2012, 4, 1024-1057.

12. D. W. Brown, T. H. Adams and N. P. Keller, Proc Natl Acad Sci U S A, 1996, 93, 14873-14877.

13. Y. N. Yin, L. Y. Yan, J. H. Jiang and Z. H. Ma, J Zhejiang Univ Sci B, 2008, 9, 787-792.

14. R. Makhuvele, K. Naidu, S. Gbashi, V. C. Thipe, O. A. Adebo and P. B. Njobeh, Heliyon, 2020, 6, e05291.

15. R. Nischitha, M. M. Vasanthkumari, B. E. Kumaraswamy and M. B. Shivanna, 3 Biotech, 2020, 10, 300.

16. E. A. Palombo, Evid Based Complement Alternat Med, 2011, 2011, 680354.

17. D. A. Khan, S. D. A. Hamdani, S. Iftikhar, S. Z. Malik, N. S. Zaidi, A. Gul, M. M. Babar, M. Ozturk, B. Turkyilmaz Unal and T. Gonenc, J Biomol Struct Dyn, 2021, 1-17.

18. S. Tiwari and J. Shankar, 3 Biotech, $2018,8,47$.

19. S. K. Shishodia, S. Tiwari and J. Shankar, Mycology, 2019, 10, 151-165.

20. J. Shankar, S. Tiwari, S. K. Shishodia, M. Gangwar, S. Hoda, R. Thakur and P. Vijayaraghavan, Front Cell Infect Microbiol, $2018,8,180$.

21. S. Tiwari, S. K. Shishodia and J. Shankar, 3 Biotech, 2019, 9, 149.

22. X. Zhao, Q. Q. Zhi, J. Y. Li, N. P. Keller and Z. M. He, Toxins (Basel), 2018, 10.

23. R. Thakur and J. Shankar, Frontiers in microbiology, 2016, 7, 192-192.

24. R. Thakur and J. Shankar, Front Microbiol, 2016, 7, 192.

25. D. T. Jones, W. R. Taylor and J. M. Thornton, Comput App/ Biosci, 1992, 8, 275-282.

26. P. Retailleau, N. Colloc'h, D. Vivarès, F. Bonneté, B. Castro, M. El Hajji, J. Mornon, G. Monard and T. Prange, Acta crystallographica. Section D,

Biological crystallography, 2004, 60, 453-462.

27. F. Kiefer, K. Arnold, M. Kunzli, L. Bordoli and T. Schwede, Nucleic Acids Res, 2009, 37, D387-392.

28. G. Yang, Y. Hu, O. E. Fasoyin, Y. Yue, L. Chen, Y. Qiu, X. Wang, Z. Zhuang and S. Wang, Frontiers in Cellular and Infection Microbiology, $2018,8$.

29. R. Syed, R. Rani, Sabeena, T. A. Masoodi, G. Shafi and K. Alharbi, Bioinformation, 2012, 8, 175-180.

30. M. Aamir Qureshi and S. Javed, Journal of Biomolecular Structure and Dynamics, 2020, 38, 3144-3155.

31. X. Zhou, S. Yu, J. Su and L. Sun, Int J Mol Sci, 2016, 17, 340.

32. K. Agrawal, J. Shankar, R. Kumar and P. Verma, Journal of Environmental Science and Health, Part B, 2020, 55, 1048-1060.

33. Y. Chauhan, J. Tatnell, S. Krosch, J. Karanja, B. Gnonlonfin, I. Wanjuki, J. Wainaina and J. Harvey, Field Crops Research, $2015,178,91-99$.

34. P. P. Singh, A. K. Jaiswal, A. Kumar, V. Gupta and B. Prakash, Scientific Reports, 2021, 11, 6832.

Page $10 / 16$ 
35. B. Alshehri and M. Palanisamy, Saudi Journal of Biological Sciences, 2020, 27, 751-756.

36. T. P. Korman, J. M. Crawford, J. W. Labonte, A. G. Newman, J. Wong, C. A. Townsend and S. C. Tsai, Proc Natl Acad Sci U S A, $2010,107,6246-6251$.

37. L. A. Goldblatt, Aflatoxin; scientific background, control, and implications, Academic Press, New York, 1969.

38. R. A. Squire, Science, 1981, 214, 877-880.

39. S. P. Kale, J. W. Cary, C. Baker, D. Walker, D. Bhatnagar and J. W. Bennett, Mycol Res, 2003, 107, 831-840.

40. J. Yu, D. Bhatnagar and T. E. Cleveland, FEBS Lett, 2004, 564, 126-130.

41. G. A. Payne, W. C. Nierman, J. R. Wortman, B. L. Pritchard, D. Brown, R. A. Dean, D. Bhatnagar, T. E. Cleveland, M. Machida and J. Yu, Med Mycol, 2006, 44, S9-S11.

42. S. Tiwari, N. Gupta, U. Malairaman and J. Shankar, National Academy Science Letters, 2017, 40, 267-271.

43. N. Mahoney and R. J. Molyneux, J Agric Food Chem, 2004, 52, 1882-1889.

Figures

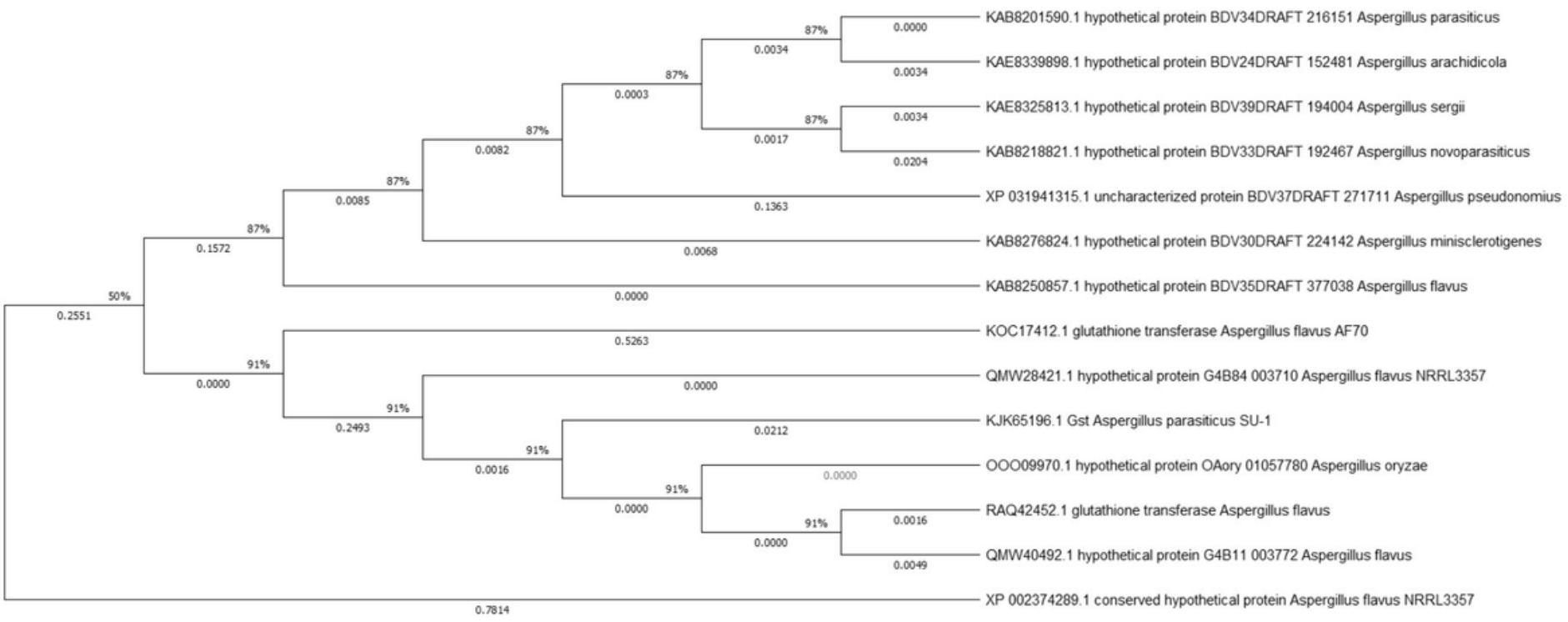

Figure 1

Phylogenetic analysis of polyketide synthase A gene of A. flavus by maximum likelihood method based on the JTT matrix-based model using MEGA 6.06 software
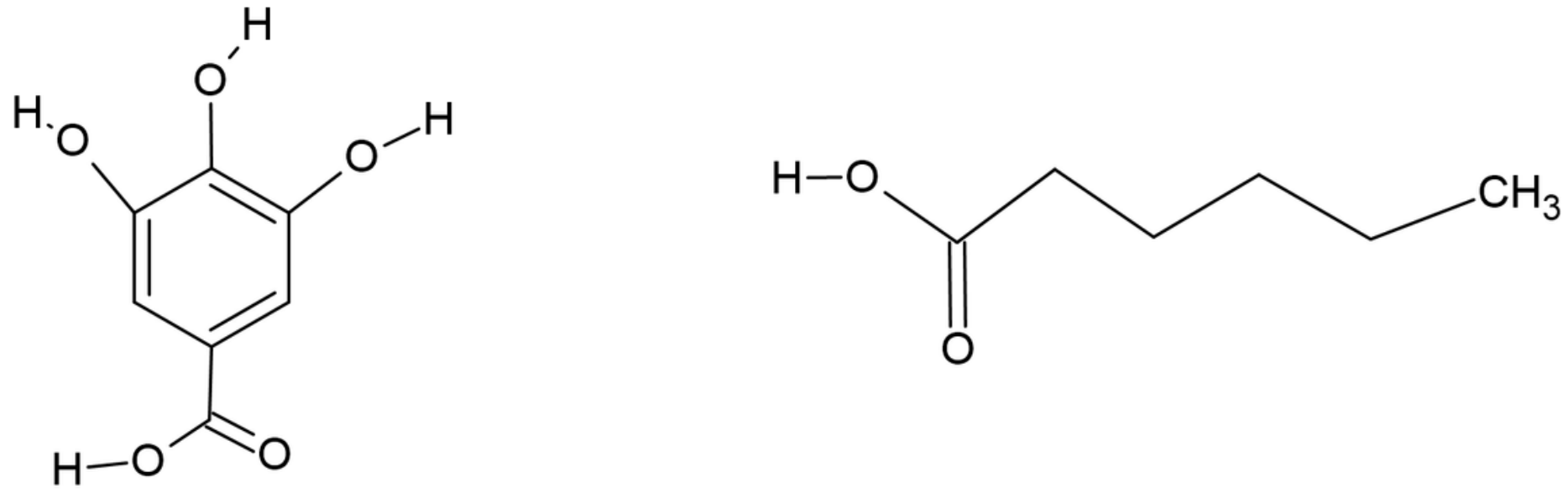
Figure 2

Three-dimensional structure of gallic Acid (PubChem CID :370) and hexanoic acid (Id: 8892) were obtained from Pubchem and drawn on Chemsketch software
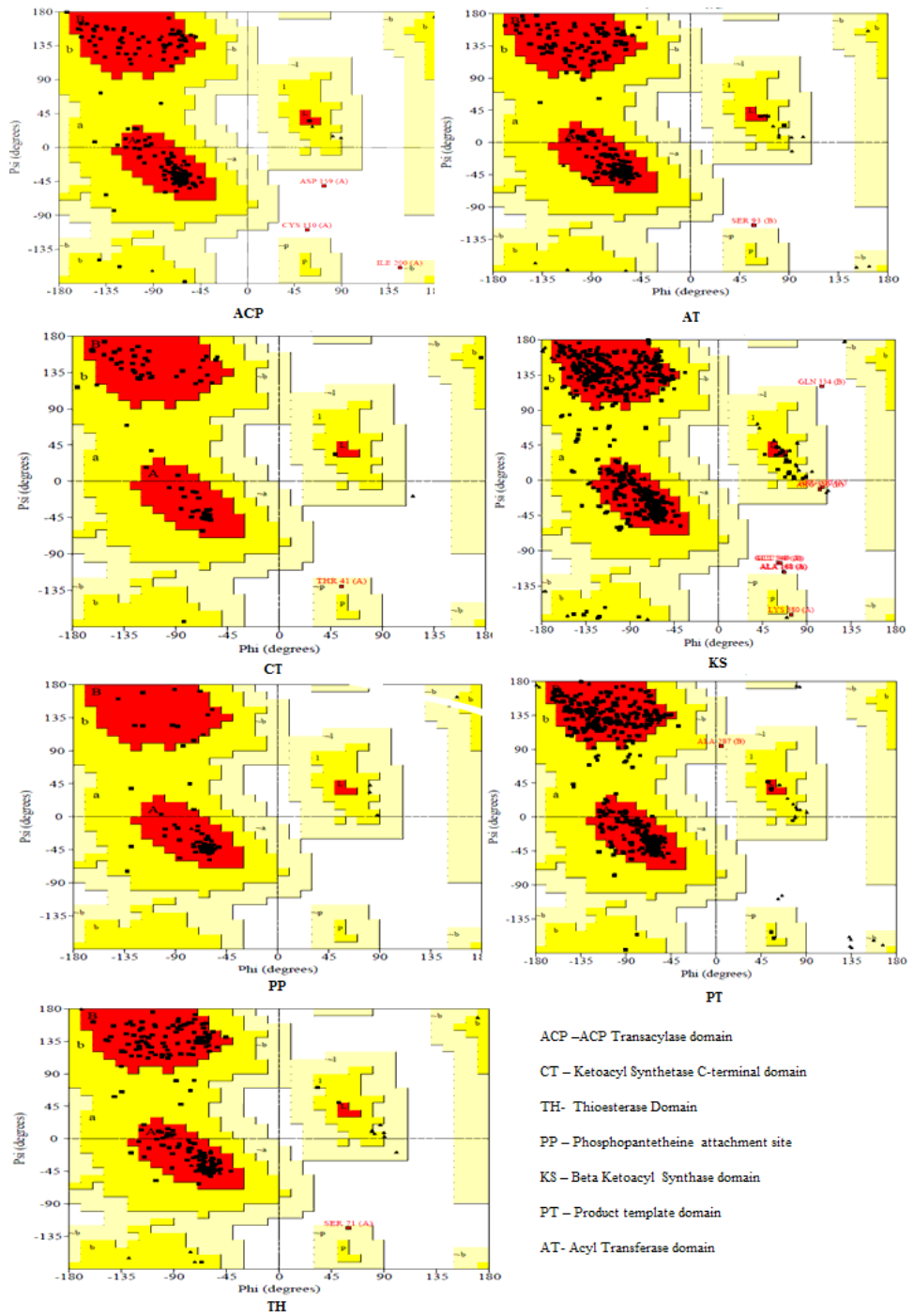

ACP -ACP Transacylase domain

CT - Ketoacyl Synthetase C-terminal domain

TH- Thioesterase Domain

PP - Phosphopantetheine attachment site

KS - Beta Ketoacyl Synthase domain

PT - Product template domain

AT- Acyl Transferase domain

Figure 3

Structure stability validated by the Ramachandran plot using Procheck software. 
Comparative analysis of binding energy

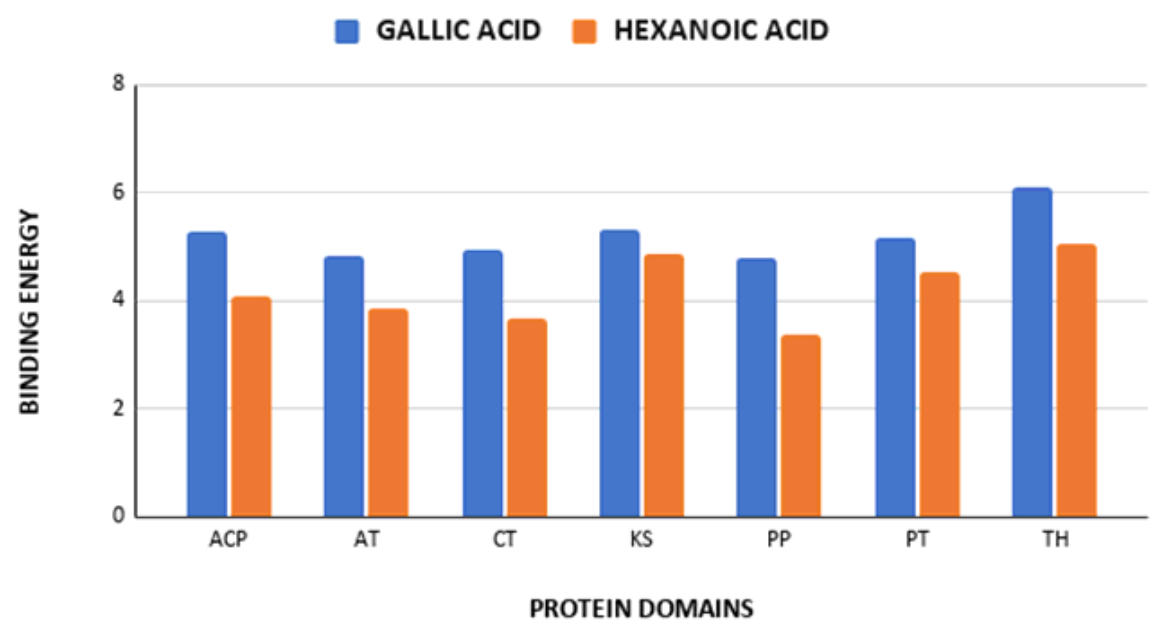
Abbreviation used:
ACP -ACP Transacylase domain
CT - Ketoacyl Synthetase C-terminal domain
TH- Thioesterase Domain
PP - Phosphopantetheine attachment site
KS - Beta Ketoacyl Synthase domain
PT - Product template domain
AT- Acyl Transferase domain

\section{Figure 4}

Comparative Analysis of Binding energy. 


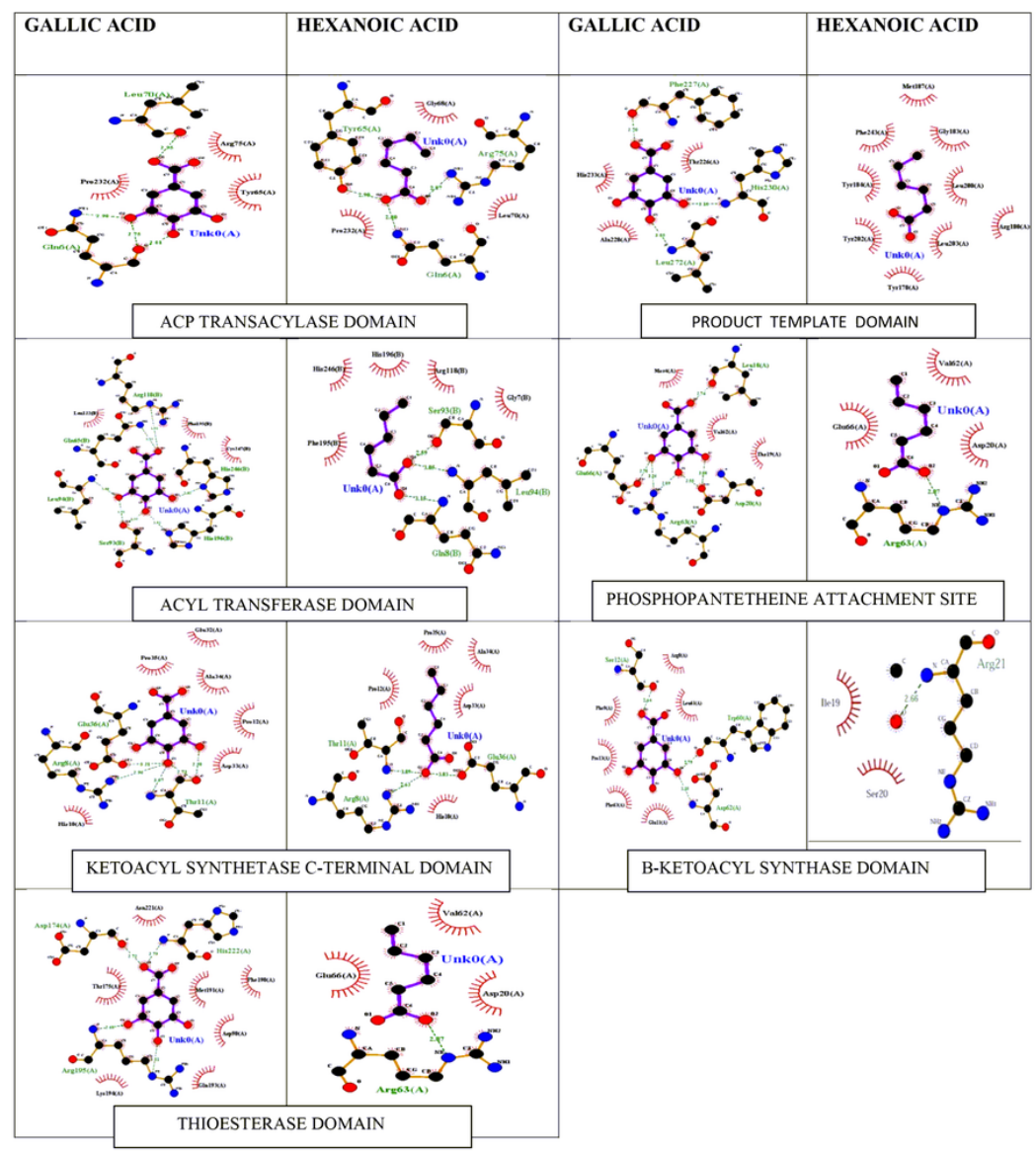

Figure 5

Post-docking correlations among active residues of seven domains of Aspergillus flavus PksA with 2 distinct ligands i.e. gallic acid and hexanoic acid, as depicted in a schematic diagram created with Ligplot. 

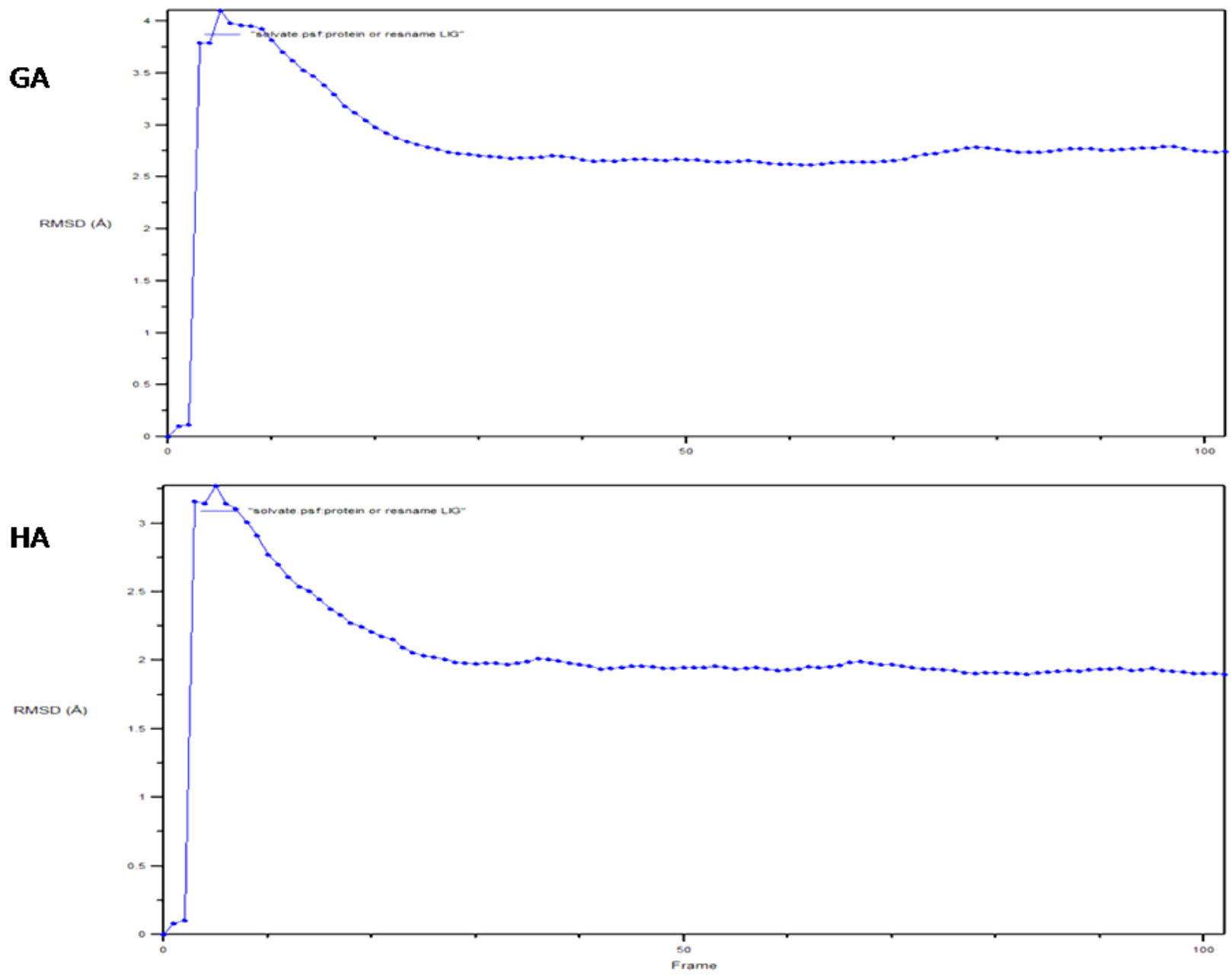

Figure 6

Comparative analysis of root mean square deviation trajectories between gallic acid (GA) and hexanoic acid(HA). 

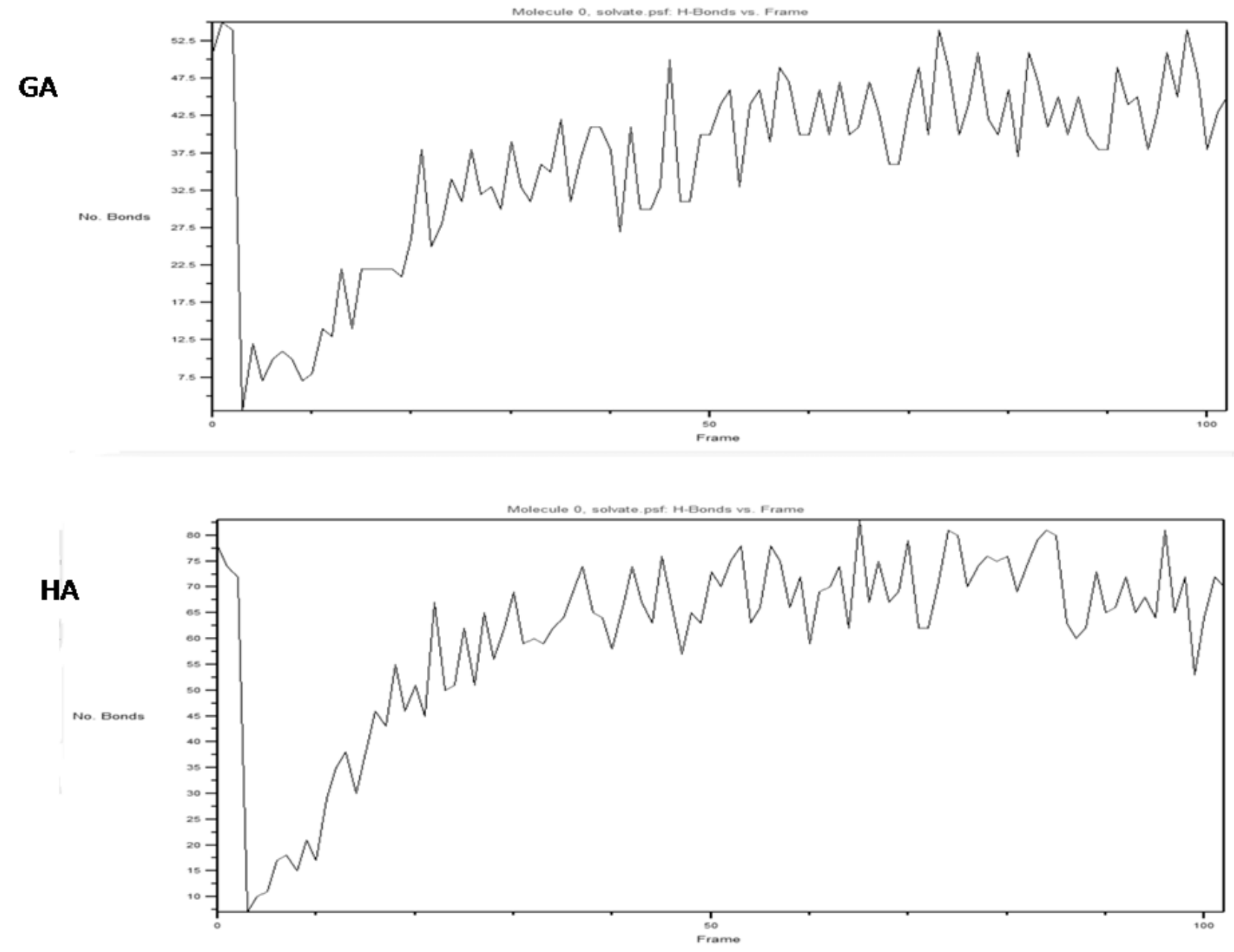

Figure 7

Hydrogen bond representation of gallic acid(GA) and hexanoic acid(HA). 